fifty-five chromosomes. In preparing Fig. $2 a$ one chromosome was unfortunately omitted although it was included in Fig. 3, the correct interpretation of the two cells. The chromosome in question is ringed on Fig. 1 here and would have been placed in group $G$ of the Denver-London system.

Although this error is most regrettable, it does not in any way alter or invalidate the argument put forward in my letter concerning the unsuitability of the Denver-London system for analysing highly abnormal chromosome constitutions.

I thank Professor P. C. Koller for bringing this mistake to my attention.

King Edward's Grammar School, Aston, Birmingham 6

Received June 29, 1971.

${ }^{1}$ Belcher, R. J., Nature, 231, 387 (1971).

\section{Variant of the Fluorescence Pattern in an Abnormal Human $Y$ Chromosome}

WILson et al. ${ }^{1}$ and Morillo-Cucci and German ${ }^{2}$ have reported on large $Y$ chromosomes with a terminal non-fluorescent segment of the long arms. We have found another variant of the fluorescence pattern in a large $Y$.

Our subject was a 36 yr old healthy man of normal body proportions, whose chromosomes were analysed because his first child, a female, had died with the clinical diagnosis of the trisomy 13 syndrome. His son, who is $4 \mathrm{yr}$ old now and clinically healthy, has the same variant $Y$ chromosome as the father: the $Y$ chromosome was consistently as large as the $D$ group chromosomes. No prominent constriction was seen in orcein-stained preparations, although some fuzziness could be demonstrated along the whole length of the long arms, as described for normal $Y$ chromosomes. Quinacrine dihydrochloride fluorescence staining of blood cultures showed a non-fluorescing gap at about the midpoint of the strongly fluorescing portion of the long $Y$ arm. This gap was consistently found in all metaphases examined (Fig. 1). The consistency of this finding in all metaphases regardless of chromosome contraction differentiates this constriction from the occasionally occurring less fluorescing gap of normal-sized $Y$ s.

The explanations put forward by Wilson and coworkers ${ }^{1}$ also apply to our cases. Either the fluorescence patterns of

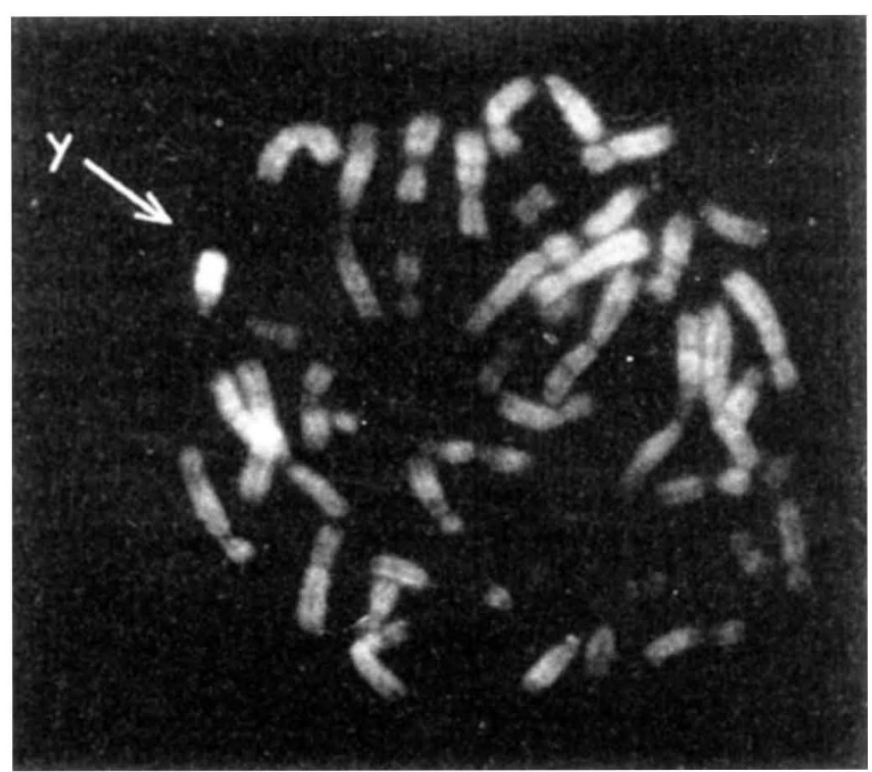

Fig. I Chromosomes showing the abnormal $Y$ with a nonfluorescing gap. long $Y$ chromosomes show variants of different kinds, or this case and other cases of abnormal $Y$ chromosomes represent examples of structural rearrangement. Morillo-Cucci and German $^{2}$ assumed a duplication of a portion of the long $Y$ arm in their case. In our case a duplication of $Y$ long arm material including a small part of the non-fluorescing portion, or, less probably, an insertion of autosomal or $X$ chromosome material into the intensely fluorescing part of the long $Y$ chromosome, would be possible explanations.

\section{ERICA M. BÜHLER \\ HANSJAKOB MÜlLER JAN MÜLLER}

Department of Genetics, GERHARD R. STALDER

\section{Children's Hospital,} University of Basle, 4000 Basle

Received July 5, 1971.

1 Wilson, M. G., Towner, J. W., Lipshin, J., and Fleisher, A., Nature, 231, 388 (1971).

${ }^{2}$ Morillo-Cucci, G., and German, J., Amer. Soc. Human Genet. Prog. (Abstract No. 37) (Indianapolis, 1970).

\section{Identification of the Trail Pheromone of a Leaf-cutting Ant, Atta texana}

THERE are numerous reports of the isolation and identification of insect sex pheromones, and recently a termite trail-following pheromone was isolated and identified ${ }^{1}$, but this is the first isolation and identification of an ant trail pheromone to be reported. We have identified the major volatile component of the trail marking substance laid down by the town ant, Atta texana (Buckley), as methyl 4-methylpyrrole-2-carboxylate (I). The worker ants of Atta texana, whose trail marking substance contains both volatile and nonvolatile components ${ }^{2}$, readily followed trails made by us with synthesized (I).

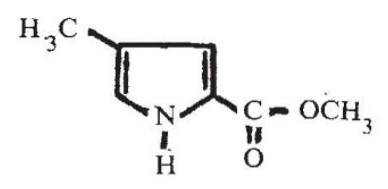

The whole bodies of $3.7 \mathrm{~kg}$ of worker ants of mixed sizes obtained from Grant Parish, Louisiana, were macerated in methylene chloride and the soluble material was distilled in a short-path still onto a condenser (cooled with dry ice) at $90^{\circ} \mathrm{C}$ and $0.05 \mathrm{~mm} \mathrm{Hg}$. The trail-following response $\mathrm{s}^{3}$ of the minor workers from a laboratory colony was used to monitor this and all subsequent isolation steps. The active nonvolatile material was saved for future study, and the volatiles were used for this investigation. The active distillate was fractionated by gas chromatography. Successive vapour phase chromatography (VPC) of the major active component on four columns (SE-30, diethylene glycol succinate, Carbowax $20 \mathrm{M}$, and silicone DC QF-1) yielded $150 \mu \mathrm{g}$ of a single compound. This very potent trail pheromone, when rechromatographed on the columns, gave a single symmetric peak on each column. Four other VPC fractions, which have not been purified or identified, are active at about ten times higher concentrations than the major active compound and are present in about five to twenty times smaller amounts.

On the basis of mass, infrared, and nuclear magnetic resonance spectra, we assigned structure (I) to the pheromone, which was confirmed by congruence of these data and of VPC retention times with those properties of an authentic synthetic sample 4 . 